\title{
Efficient generation of All Regular Non-Dominated Coteries*
}

\author{
Kazuhisa Makino \\ Department of Systems and Human Science \\ Graduate School of Engineering Science \\ Osaka University \\ Toyonaka, Osaka, 560-8531, Japan \\ makino@sys.es.osaka-u.ac.jp
}

\author{
Tiko Kameda \\ School of Computing Science \\ Faculty of Applied Sciences \\ Simon Fraser University \\ Burnaby, British Columbia, V5A 1S6, Canada \\ tiko@cs.sfu.ca
}

\begin{abstract}
A coterie is a family of subsets such that every pair of subsets in it has at least one element in common but neither is a subset of the other. We introduce an operator $\sigma$, which transforms a ND (non-dominated; see the Introduction for definition) coterie to another ND coterie. A "regular" coterie is a natural generalization of a "vote-assignable" coterie, which is used in some practical applications. We show that any regular ND coterie $C$ can be transformed to any other regular ND coterie $D$ by judiciously applying $\sigma$ operations to $C$ at most $|C|+|D|-2$ times.

As another application of the $\sigma$ operation, we present an incrementally-polynomial-time algorithm for generating all regular ND coteries. We then introduce the concept of a "g-regular" function, as a generalization of availability. We show how to construct an optimum coterie $C$ with respect to a g-regular function in $O\left(n^{3}|C|\right)$ time.
\end{abstract}

\section{INTRODUCTION}

A coterie $C$ under an underlying set $U=\{1,2, \ldots, n\}$ is a family of subsets (called quorums) of $U$ satisfying the intersection property (i.e., for any pair $S, R \in C, S \cap R \neq \emptyset$ holds), and minimality (i.e., no quorum in $C$ contains any other quorum in $C$ ) $[8,12]$. The concept of a coterie has applications in diverse areas (see e.g., $[6,8,12,15]$ ).

For example, to achieve mutual exclusion in a distributed system, let the elements in $U$ represent the sites in the distributed system. A task is allowed to enter a critical section only if it can get permissions from all the members of a quorum $Q \in C$, where each site is allowed to issue at most

\footnotetext{
${ }^{*}$ This research was partially supported by the Scientific Grants in Aid by the Ministry of Education, Science, Sports and Culture of Japan.
}

one permission at a time. By the intersection property, it is guaranteed that at most one task can enter the critical section at any time.

A coterie $D$ is said to dominate another coterie $C$ if, for $\forall Q \in C$, there exists a quorum $Q^{\prime} \in D$ satisfying $Q^{\prime} \subseteq Q$ [8]. A coterie $C$ is non-dominated (ND) if no other coterie dominates it. ND coteries are important in practical applications, since they have maximal "efficiency" in some sense $[3,8]$.

Given a family $C$ of subsets of $U$, which is not necessarily a coterie, we define a positive (i.e., monotone) Boolean function $f_{C}$ such that $f_{C}(x)=1$ if the Boolean vector $x \in\{0,1\}^{n}$ is greater than or equal to the characteristic vector of some subset $^{1}$ in $C$, and 0 otherwise. It was shown in [10] that $C$ is a coterie (resp., ND coterie) if and only if $f_{C}$ is dualminor (resp., self-dual) [14]. Based on this characterization, Boolean algebra can be exploited to derive various properties of (ND) coteries.

A coterie $C$ is said to be vote-assignable if there exist a vote assignment $w: U \mapsto \mathbb{R}^{+}$and a threshold $t \in \mathbb{R}^{+}$such that $w(S) \geq t$ if and only if $S \supseteq Q$ for some $Q \in C[8,9$, $18]$, where $\mathbb{R}^{+}$is the set of nonnegative real numbers and $w(S)=\sum_{i \in S} w(i)$. It is easy to see that there is a one-toone correspondence between vote-assignable coteries (resp., ND coteries) $C$ and dual-minor (resp., self-dual) threshold Boolean functions $f_{C}$ (see Section 2). The vote-assignable coteries are important and have been used in many practical applications, since they can be handled efficiently (see e.g., $[8,9,18,19])$. We assume in this paper that a vote assignment $w$ satisfies $w(i) \geq w(j)$ for all $i<j$, since we are interested in coteries which are non-equivalent under permutation on $U$. A coterie $C$ is equivalent to a coterie $C^{\prime}$ under permutation, if $C$ can be transformed into $C^{\prime}$ by permuting the elements of $U$. A coterie $C$ is said to be regular if, for every $Q \in C$ and every pair $(i, j) \in U \times U$ with $i<j, i \notin Q$ and $j \in Q$, there exists quorum $Q^{\prime} \in C$ such that $Q^{\prime} \subseteq(Q \backslash\{j\}) \cup\{i\} .^{2} \quad$ By definition, a vote-

\footnotetext{
${ }^{1}$ The $i$ th component of the characteristic vector is $1(0)$ if $i \in U$ is (not) contained in the subset.

${ }^{2}$ This definition was motivated by the definition of regular Boolean functions. See Section 2.3.
} 
assignable coterie $C$ is always regular, though the converse is not true in general. It is known that most regular coteries are vote-assignable [14], in particular, all regular ND coteries for $n \leq 9$ are vote-assignable.

Among the important problems regarding coteries are:

(i) construct "optimal" ND coteries according to a certain criterion, such as availability and load (equivalently, construct an "optimal" positive self-dual function), and

(ii) generate all ND coteries (equivalently, all positive selfdual functions) systematically.

As for (i), let us consider the availability of a coterie. Assume that element $i$ is operational with probability $p_{i}$, where the probabilities for different components are independent. Given the operational probabilities $p_{i}, i \in U$, where we assume without loss of generality that

$$
1 \geq p_{1} \geq p_{2} \geq \ldots \geq p_{n} \geq 0
$$

(if not, we permute the elements so that (1) holds), the availability of a coterie $C$ is the probability that the set of operational elements contains at least one quorum in $C$. Availability is clearly an important concept in practical applications, and it is desirable to construct a coterie with the maximum availability.

The availability of coteries has been studied extensively. It is known $[1,17]$ that the elements $i \in U$ with $p_{i}<1 / 2$ can be ignored, i.e., there exists a maximum-availability coterie $C$ such that no quorum in $C$ contains $i$. (In the case where $p_{i}<$ $1 / 2$ holds for all $i, C=\{\{1\}\}$ has the maximum availability $[1,7,16])$. Thus, we shall assume that

$$
p_{1} \geq p_{2} \geq \cdots \geq p_{n} \geq 1 / 2 .
$$

It is also known that, if either $p_{1}=1$ or $p_{1} \leq 1 / 2$, then $C=$ $\{\{1\}\}$ has the maximum availability. If $1 \neq p_{1}>1 / 2$, on the other hand, it is demonstrated in $[17,19]$ that the coterie $C_{\text {max }}$, given below, maximizes availability. First define the weight for $i \in U$ by

$$
w^{*}(i)=\log _{2}\left(p_{i} /\left(1-p_{i}\right)\right),
$$

and introduce the notation $w^{*}(S)=\sum_{i \in S} w^{*}(i)$ for $S \subseteq U$. Now, $Q \in C_{\max }$ if

(a) $w^{*}(Q)\left(=w^{*}(U \backslash Q)\right)=w^{*}(U) / 2$ and $1 \in Q(1$ is an element of $U)$, or

(b) $Q$ is a minimal subset of $U$ with $w^{*}(Q)>w^{*}(U) / 2$, and $Q$ does not contain any quorum of type (a).

Since this coterie $C_{\max }$ is vote-assignable, $[1,17,19]$ proposed algorithms to compute a vote assignment $w$ from $w^{*}$, called tie-breaking, in order to remove case (a). An exponential algorithm is proposed in [19] to find the "optimal" tie-breaking rule, while $[1,17]$ present polynomial-time approximation algorithms for it. The main problem with the above definition of $C_{\max }$ is that there may exist a subset $S \subseteq U$ such that $w^{*}(S)=w^{*}(U \backslash S)$ (case (a)), because of which a simple vote assignment $w$ (showing that $C_{\max }$ is vote-assignable) is not easily obtainable, and that the weight $w^{*}(i)$ is, in general, not a rational number, hence we cannot compute $w^{*}(S)=\sum_{i \in S} w^{*}(i)$ in polynomial time. For the above reasons, no polynomial algorithm for constructing a maximum-availability coterie was known. In this paper, we present a polynomial-time algorithm for it. More precisely, we define a "g-regular" function as a generalization of availability (see Section 5), and then show that, given a g-regular function $\Phi$, we can compute a coterie $C$ which maximizes $\Phi$ in $O\left(n^{3}|C|\right)$ time, where $|C|$ is the number of quorums in $C$.

Problem (ii) is known to be useful to solve (i) $[5,8]$. To solve (i), one might first enumerate all (or some) ND coteries efficiently, and select the best one under a certain criterion, which may not be easily computable. This procedure is useful when $n$ is small, or when we have enough time to compute it.

The generation of all ND coteries in a certain subclass of vote-assignable ND coteries was discussed in [14], which is used to give a lower bound on the number of all voteassignable ND coteries. However, the procedure is not polynomial and computes a proper subclass of vote-assignable ND coteries. H. Garcia-Molina and D. Barbara [8] proposed an algorithm to generate all ND coteries in a certain superclass of regular ND coteries. However, it is also not polynomial. J. C. Bioch and T. Ibaraki [5] later came up with a polynomial time algorithm to generate all ND coteries, and compiled a list containing all ND coteries under up to 7 elements, which are non-equivalent under permutation. We remark here that their algorithm is not polynomial, if equivalent duplicates are to be deleted from the output. In fact, they compiled a list of all ND coteries under up to 7 elements by first running their algorithm and then selecting non-equivalent representatives from among them. In this paper, we present a polynomial algorithm to generate all regular ND coteries. Since no regular ND coterie $C$ is equivalent to any other regular ND coterie $C^{\prime}(\neq C)$ under permutation, our algorithm does not output ND coteries which are equivalent under permutation. Although our algorithm outputs only regular ND coteries, it is practically useful, because all ND coteries under up to $n=5$ elements are all regular (if we consider their representatives), and when $n$ is relatively is small, a large fraction of ND coteries are regular [14]. Moreover, if the objective function of problem (i) is g-regular (e.g., the availability of a coterie), then we can restrict our attention to regular coteries.

After defining necessary terminologies in Section 2 we discuss in Section 3 two operations, called $\rho$ and $\sigma$, which transform a positive self-dual function $f$ (representing a ND coterie) into another positive self-dual function (representing another ND coterie), by making a minimal change in the set of minimal true vectors of $f$.

Section 4 shows that any regular self-dual function $f$ (representing a regular ND coterie) can be transformed into any other regular self-dual function $g$ (representing any other regular ND coterie) by judiciously applying $\sigma$ operations to $f$ at most $|\min T(f)|+|\min T(g)|-2$ times. In Sections 5 and 6 , we consider the problems of computing an optimal self-dual function with respect to a g-regular functional $\Phi$ and generating all regular self-dual functions, as applications 
of the above transformation.

In addition to the theory of coteries, the concepts of selfduality and regularity play important roles in diverse areas such as learning theory, operations research and set theory. The results of this paper are relevant to all these areas.

Due to the space limitation, the proofs of some results are omitted (see [13]).

\section{PRELIMINARIES}

A Boolean function (a function in short) is a mapping $f$ : $\{0,1\}^{n} \mapsto\{0,1\}$, where $v \in\{0,1\}^{n}$ is called a Boolean vector (a vector in short). If $f(v)=1$ (resp., 0), then $v$ is called a true (resp., false) vector of $f$. The set of all true vectors (resp., false vectors) of $f$ is denoted by $T(f)$ (resp., $F(f))$. For any two functions $f$ and $g$, we say that $f$ is covered by $g$ (written $f \leq g$ ) if $T(f) \subseteq T(g)$. For a vector $v=\left(v_{1}, v_{2}, \ldots, v_{n}\right)$, we define $O N(v)=\left\{j \mid v_{j}=1\right\}$ and $O F F(v)=\left\{j \mid v_{j}=0\right\}$.

The argument $x$ of function $f$ is represented as a vector $x=\left(x_{1}, x_{2}, \ldots, x_{n}\right)$, where each $x_{i}$ is a Boolean variable. A variable $x_{i}$ is said to be relevant if there exist two vectors $v$ and $w$ such that $f(v) \neq f(w), v_{i} \neq w_{i}$, and $v_{j}=w_{j}$ for all $j \neq i$; otherwise, it is said to be irrelevant. The set of all relevant variables of a function $f$ is denoted by $V_{f} \subseteq V=\left\{x_{1}, x_{2}, \ldots, x_{n}\right\}$. A literal is either a variable $x_{i}$ or its complement $\bar{x}_{i}$. A term $t$ is a conjunction $\bigwedge_{i \in P(t)} x_{i} \wedge$ $\bigwedge_{j \in N(t)} \bar{x}_{j}$ of literals such that $P(t), N(t) \subseteq\{1,2, \ldots, n\}$ and $P(t) \cap N(t)=\emptyset$; E.g., $t_{1}=x_{1} x_{4} \bar{x}_{5} x_{6}$ is a term, while $t_{2}=x_{2} x_{4} \bar{x}_{2}$ is not. A disjunctive normal form (DNF) is a disjunction of distinct terms. It is easy to see that any function $f$ can be represented in DNF, whose variable set is $V_{f}$.

We sometimes do not distinguish a formula (e.g., DNF) from the function it represents, if no confusion arises.

\subsection{Positive functions}

For a pair of vectors $v, w \in\{0,1\}^{n}$, we write $v \leq w$ if $v_{j} \leq w_{j}$ holds for all $j \in V$, and $v<w$ if $v \leq w$ and $v \neq w$, where we define $0<1$. For a set of vectors $S \subseteq\{0,1\}^{n}$, $\min _{\geq} S$ (resp., $\max _{\geq} S$ ) denotes the set of all minimal (resp., maximal) vectors in $S$ with respect to $\geq$; For example, for a function $f, \min _{\geq} T(f)$ (resp., $\max _{\geq} F(f)$ ) denotes the set of all minimal true vectors (resp., maximal false vectors) of $f$. We sometimes use $\min S$ (resp., $\max S$ ) instead of $\min _{\geq} S$ (resp., $\max _{\geq} S$ ), if no confusion arises. A function $f$ is said to be positive or monotone if $v \leq w$ always implies $f(v) \leq f(w)$. A prime implicant of a function $f$ is a term $t$ that implies $f$ but no proper subterm of $t$ implies $f$. There is a one-to-one correspondence between $\min T(f)$ and the set of all prime implicants of $f$, such that a vector $v$ corresponds to the term $t_{v}$ defined by $t_{v}=x_{i_{1}} x_{i_{2}} \cdots x_{i_{k}}$ if $v_{i_{j}}=1, j=$ $1,2, \ldots, k$ and $v_{i}=0$ otherwise. For example, the vector $v=(1010)$ corresponds to the term $t_{v}=x_{1} x_{3}$. We also use the notation $t_{\bar{v}}$ to denote the term $x_{j_{1}} x_{j_{2}} \cdots x_{j_{l}}$, where $\left\{j_{1}, j_{2}, \ldots, j_{l}\right\}=\{1,2, \ldots, n\} \backslash\left\{i_{1}, i_{2}, \ldots, i_{k}\right\}$. For the above $v=(1010)$, we have $t_{\bar{v}}=x_{2} x_{4}$.

It is known that a positive function $f$ has the unique mini- mal disjunctive normal form (MDNF), consisting of all the prime implicants of $f$, where $N(t)=\emptyset$ for each prime implicant $t$. In this paper, we sometimes represent the MDNF of a positive function such as $f=x_{1} x_{2}+x_{2} x_{3}+x_{3} x_{1}$ by a simplified form $f=12+23+31$, by using only the subscripts of the literals. The set of minimal true vectors of this function is $\min T(f)=\{(110),(011),(101)\}$, if $f$ is a 3 -variable function. Coteries can be conveniently modeled by positive Boolean functions, based on the fact that min $T(f)$ can represent a family of subsets, none of which includes the other [10]. For example, the above $\min T(f)$ represents a coterie $C=\{\{1,2\},\{2,3\},\{3,1\}\}$.

\subsection{Dual-comparable functions}

The dual of a function $f$, denoted $f^{d}$, is defined by

$$
f^{d}(x)=\bar{f}(\bar{x}),
$$

where $\bar{f}$ and $\bar{x}$ denote the complement of $f$ and $x$, respectively. As is well-known, $f^{d}$ is obtained from $f$ by interchanging $+(\mathrm{OR})$ and $\cdot(\mathrm{AND})$, as well as the constants 0 and 1. It is easy to see that $(f+g)^{d}=f^{d} g^{d},(f g)^{d}=f^{d}+g^{d}$, and so on. A function is called dual-minor if $f \leq f^{d}$, dualmajor if $f \geq f^{d}$ and self-dual if $f=f^{d}$. For example, $f=123$ is dual-minor since $f^{d}=1+2+3$ satisfies $f \leq f^{d}$.

If $f$ is positive, then $f^{d}$ is also positive. In this case, an alternative definition of $f^{d}$ is given by the condition that $v \in T\left(f^{d}\right)$ if and only if $v$ is a transversal of $\min T(f)$; i.e., it satisfies $O N(v) \cap O N(w) \neq \emptyset$ for all $w \in \min T(f)$.

Let $\mathcal{C}_{S D}(n)$ (resp., $\mathcal{C}_{D M A}(n)$ and $\left.\mathcal{C}_{D M I}(n)\right)$ denote the class of all positive self-dual (resp., dual-major and dual-minor) functions of $n$ variables. Note that in these definitions functions may have some irrelevant variables.

\subsection{Regular, 2-monotonic and threshold func- tions}

A positive function $f$ is said to be regular if, for every $v \in$ $\{0,1\}^{n}$ and every pair $(i, j)$ with $i<j, v_{i}=0$ and $v_{j}=1$, the following condition holds:

$$
f(v) \leq f\left(v+e^{(i)}-e^{(j)}\right),
$$

where $e^{(k)}$ denotes the unit vector which has a 1 in its $k$-th position and 0's in all other positions.

In order to define an important partial order on $\{0,1\}^{n}$, we first define the concept of the profile of a vector $v \in\{0,1\}^{n}$ as follows:

$$
\operatorname{prof}_{v}(k)=\sum_{j \leq k} v_{j},
$$

where $k=1,2, \ldots, n$. If $v, w \in\{0,1\}^{n}$, where $v \neq w$, satisfy $\operatorname{prof}_{v}(k) \leq \operatorname{prof}_{w}(k)$ for all $k$, then we write $v \prec w$ (or $w \succ v$ ), and we say that $v$ supports $w$. If $v \prec w$ or $v=w$, then we write $v \preceq w$ (or $w \succeq v$ ).

It is clear from the above definition that $v \prec w$ if and only if $\bar{v} \succ \bar{w}$, since $\operatorname{prof}_{\bar{v}}(k)=k-\operatorname{prof}_{v}(k)$. Note that $v \leq$ $w$ implies $v \preceq w$ but the converse is not always true. $\overline{\mathrm{A}}$ function $f$ is said to be profile-monotone if $v \prec w$ implies $f(v) \leq f(w)$. The following lemma is proved in [14]. 
LEMma 1 ([14]). A function $f$ is regular if and only if $f$ is profile-monotone.

For a set of vectors $S \subseteq\{0,1\}^{n}, \min _{\succeq} S$ (resp., $\max _{\succeq} S$ ) denotes the set of all minimal (resp., maximal) vectors in $S$ with respect to $\succeq$. For any set of vectors $S \subseteq\{0,1\}^{n}$, we have $\min _{\succeq} S \subseteq \min S\left(=\min _{\geq} S\right)$ and $\max _{\succeq} S \subseteq \max S(=$ $\max >S)$, since $v \geq w$ implies $v \succeq w$. It follows from Lemma 1 that a regular function $f$ is uniquely determined by $\min _{\succeq} T(f)$.

A positive function $f$ is called 2-monotonic if there exists a linear ordering on $V$, for which $f$ is regular. The 2monotonicity was originally introduced in conjunction with threshold functions (e.g., [14]), where a positive function $f$ is a threshold function if there exist $n$ nonnegative real numbers (weights) $w_{1}, w_{2}, \ldots, w_{n}$ and a non-negative real number (threshold) $t$ such that:

$$
f(x)=1 \text { if and only if } \sum w_{i} x_{i} \geq t .
$$

As this $f$ satisfies (2) by permuting variables so that $w_{i}>w_{j}$ implies $i<j$, a threshold function is always 2-monotonic, although the converse is not true [14].

\section{THE OPERATORS $\rho$ AND $\sigma$}

Let $f$ be a positive function of $n$ variables. Throughout this paper, we assume that $f$ is nontrivial in the sense that $f \not \equiv 0,1$ and $n \geq 1$. Given a vector $v \in \min T(f)$, the operation $\rho_{v}$ applied to $f$ removes $v$ from $T(f)$ and then adds $\bar{v}$ to $T(f)[5]$. More precisely, while adding $\bar{v}$, all the vectors larger than $\bar{v}$ are also added to $T(f)$. Therefore,

$$
T\left(\rho_{v}(f)\right)=(T(f) \backslash\{v\}) \cup T_{\geq}(\bar{v}),
$$

where $T_{\geq}(\bar{v})=\left\{w \in\{0,1\}^{n} \mid w \geq \bar{v}\right\}$. An equivalent definition is

$$
\rho_{v}(f)=f_{\backslash v}+t_{\bar{v}}+t_{v} t_{\bar{v}}^{d}
$$

where $f_{\backslash v}$ denotes the function defined by all the prime implicants of $f$ except $t_{v}$, and $t_{v}^{d}$ denotes the dual of $t_{\bar{v}}$. We note that, if $t_{v}=x_{i_{1}} x_{i_{2}} \cdots x_{i_{k}}$ and $t_{\bar{v}}=x_{j_{1}} x_{j_{2}} \cdots x_{j_{l}}$, then

$$
t_{v} t \frac{d}{v}=x_{i_{1}} x_{i_{2}} \cdots x_{i_{k}}\left(x_{j_{1}}+x_{j_{2}}+\cdots+x_{j_{l}}\right)
$$

represents all the vectors larger than $v$. The expression (5) is not necessarily in MDNF, even if $f_{\backslash v}$ is represented by its MDNF, because some of the prime implicants in $t_{\bar{v}}+t_{v} t \frac{d}{v}$ may cover or may be covered by some prime implicants of $f_{\backslash v}$.

Given a vector $v \in \min T(f)$ and a variable set $I$ with $V_{f} \subseteq$ $I \subseteq V$, we define the operation $\sigma_{(v ; I)}$ by

$$
\sigma_{(v ; I)}(f)=f_{\backslash v}+t_{\overline{v[I]}}+t_{v[I]} t \frac{d}{v[I]},
$$

where $v[I]$ denotes the projection of $v$ on $I$; e.g., if $v=$ (1100), $I_{1}=\{1,2,3\}$ and $I_{2}=\{2,3\}$, then $v\left[I_{1}\right]=(110)$ and $v\left[I_{2}\right]=(10)$. By definition, $\sigma_{(v ; V)}=\rho_{v}$ holds. This operation $\sigma_{(v ; I)}$ is implicitly used in [8]. ables,

EXAMPLE 1. Consider a positive function of $n=7$ vari-

$$
f=12+13+145+234+235 \text {. }
$$

For this function, we have $V_{f}=\left\{x_{1}, x_{2}, x_{3}, x_{4}, x_{5}\right\}$. For $v=(0111000)$, we show below how operations $\rho$ and $\sigma$ are applied:

$$
\begin{aligned}
\rho_{v}(f)= & 12+13+145+235+1567 \\
& +234(1+5+6+7) \\
= & 12+13+1567+2346+2347+235, \\
\sigma_{\left(v ; V_{f}\right)}(f)= & 12+13+145+235+15+234(1+5) \\
= & 12+13+15+235 .
\end{aligned}
$$

Let $f$ be a function on the variable set $V=\{1,2, \ldots, n\}$. For a variable set $I \subseteq V$, the projection of $f$ on $I$, denoted by $\operatorname{Proj}_{I}(f)$, is the function on $I$ obtained from $f$ by fixing $x_{i}=0$ for all $x_{i} \in V \backslash I$, i.e.,

$$
\operatorname{Proj}_{I}(f)\left(x_{1}, x_{2}, \ldots, x_{|I|}\right)=f\left(x_{1}, x_{2}, \ldots, x_{|I|}, 0, \ldots 0\right),
$$

if $I=\left\{x_{1}, x_{2}, \ldots, x_{|I|}\right\}$. For a variable set $J \supseteq V$, the expansion of $f$ to $J$, denoted by $\operatorname{Exp}_{J}(f)$, is the function on $J$ obtained from $f$ by adding irrelevant variables $x_{i} \in J \backslash V$. By definition, $f$ and its expansion can be represented by the same MDNF. Since $I \supseteq V_{f}$, we have

$$
\sigma_{(v ; I)}(f)=\operatorname{Exp}_{V}\left(\rho_{v[I]}\left(\operatorname{Proj}_{I}(f)\right)\right) .
$$

Thus $\sigma$ has properties similar to those of $\rho$.

Now, for a specified class $\mathcal{C}(n)$ of positive functions of $n$ variables, we say that $\rho$ (resp., $\sigma)$ preserves $\mathcal{C}(n)$ if $\rho_{v}(f) \in \mathcal{C}(n)$ holds for all $f \in \mathcal{C}(n)$ and $v \in \min T(f)$ (resp., $\sigma_{(v ; I)}(f) \in$ $\mathcal{C}(n)$ holds for all $f \in \mathcal{C}(n), v \in \min T(f)$ and $\left.I \subseteq V_{f}\right)$.

THEOREM 1. The operations $\rho$ and $\sigma$ defined above preserve the classes $\mathcal{C}_{S D}(n), \mathcal{C}_{D M A}(n)$ and $\mathcal{C}_{D M I}(n)$.

Proof. This theorem is proved for $\rho$ in [5]. Consider any function $f \in \mathcal{C}_{S D}(n)$ and any set $I$ satisfying $V_{f} \subseteq I \subseteq V$. We have $\operatorname{Proj}_{I}(f) \in \mathcal{C}_{S D}(|I|)$, and hence $\rho_{v}\left(\operatorname{Proj}_{I}(\bar{f})\right) \in$ $\mathcal{C}_{S D}(|I|)$ by the above-cited result in [5]. It is clear that, for any $g \in \mathcal{C}_{S D}(|I|)$, we have $\operatorname{Exp}_{V}(g) \in \mathcal{C}_{S D}(n)$. Thus by $(5), \sigma_{(v ; I)}$ preserves $\mathcal{C}_{S D}(n)$. Similarly for $\mathcal{C}_{D M A}(n)$ and $\mathcal{C}_{D M I}(n)$.

Let us further note that, if $f$ is self-dual, then $\rho_{v}(f), v \in$ $\min T(f)$, is specified simply by

$$
T\left(\rho_{v}(f)\right)=(T(f) \backslash\{v\}) \cup\{\bar{v}\},
$$

i.e., by interchanging $v$ with $\bar{v}$ in $T(f)$ [5]. To see the effect of $\sigma_{(v ; I)}$ on $T(f)$, where $V_{f} \subseteq I \subseteq V$, define

$$
v[I]_{\underline{*}}=\left\{u \in\{0,1\}^{n} \mid u[I]=v[I]\right\} .
$$

It is easy to see that

$$
T\left(\sigma_{(v ; I)}(f)\right)=\left(T(f) \backslash v[I]_{\underline{*}}\right) \cup \bar{v}[I]_{\underline{*}} .
$$

Now consider a sequence of transformations from a positive self-dual function $f$ to another positive self-dual function $g$,

$$
\begin{aligned}
& f_{0}(=f) \longrightarrow f_{1} \longrightarrow \ldots \longrightarrow f_{m_{1}}(=g), \\
& g_{0}(=f) \longrightarrow g_{1} \longrightarrow \ldots \longrightarrow g_{m_{2}}(=g) \text {, }
\end{aligned}
$$


where $f_{i+1}=\rho_{v^{(i)}}\left(f_{i}\right), v^{(i)} \in \min T\left(f_{i}\right), g_{i+1}=\sigma_{\left(w^{(i)} ; I_{i}\right)}\left(g_{i}\right)$, $w^{(i)} \in \min T\left(g_{i}\right)$, and $I_{i} \supseteq V_{g_{i}}$. We can see that $m_{1}, m_{2} \geq$ $|\min T(f) \backslash \min T(g)|$ and $m_{1} \geq|T(f) \backslash T(g)|$. The latter implies that $m_{1}$ might be exponential in $n$ and $\min T(f)$, while $m_{2}$ might be small. In the next section, we consider $\rho$ and $\sigma$ operations on regular self-dual functions, and give a transformation algorithm between two regular self-dual functions $f$ and $g$, which satisfies

$$
m_{2} \leq|\min T(f)|+|\min T(g)|-2 .
$$

\section{TRANSFORMATION OF REGULAR SELF-DUAL FUNCTIONS}

The goal of this section is to present an efficient algorithm, TRANS-REG-SD, which transforms a given regular selfdual function $f$ to the one-variable regular self-dual function $g=x_{1}$. It applies a sequence of $\sigma$ operations to $f$, generating a sequence of regular self-dual functions in the process. As we will show, this algorithm can be used to transform a given regular self-dual function of $n$ variables to any other regular self-dual function of $n$ variables, some of which may be irrelevant. We need to prove a number of lemmas to achieve this goal.

We start with the following lemma, which shows that $\rho_{v}$ preserves regularity if $v$ satisfies a certain condition. (We have already seen that $\rho_{v}$ preserves self-duality.) Recall that $\rho_{v}(f)$ is specified by (8), and therefore, we concentrate on the vectors $v$ and $\bar{v}$.

LEMma 2. Let $f$ be a regular self-dual function, and let $v \in \min T(f) . \rho_{v}(f)$ is regular if and only if $v \in \min _{\succeq} T(f)$ and $\bar{v} \nprec v$.

Proof. By definition, $\rho_{v}(f)$ is regular (i.e., profile-monotone) if

$$
\rho_{v}(f)(u) \leq \rho_{v}(f)(w) \text { for any } u \prec w .
$$

Let us first consider the only-if part. Recall that $\rho_{v}(f)$ can be specified by (8). Thus we have $\rho_{v}(f)(v)=0$ and $\rho_{v}(f)(\bar{v})=1$, which, together with (10), implies $\bar{v} \nprec v$. Moreover, since $v \in \min T(f)$, if $v \notin \min _{\succeq} T(f)$ then there exists a vector $u \in \min _{\succeq} T(f)$ supporting $v$, i.e., $u \prec v$. Now we have $\rho_{v}(f)(v)=0$ and $\rho_{v}(f)(u)=1$, which contradicts (10) with $w=v$. Thus $v \in \min _{\succeq} T(f)$ holds.

We now turn to the proof of the if part, and show that $\rho_{v}(f)$ is profile-monotone. Eq. (10) clearly holds if $u, w \notin$ $\{v, \bar{v}\}$, since $f$ is profile-monotone (see (8)). If $u=v$ in (10), then the lefthand side becomes $\rho_{v}(f)(v)=0$ by $(8)$, and (10) holds for any $w$. Similarly, if $w=\bar{v}$ in (10), then the righthand side becomes $\rho_{v}(f)(\bar{v})=1$ by (8), and (10) holds for any $u$. Assume now that $u=\bar{v}(\prec w)$, in which case $w \neq v$ by the condition in the lemma. Then we have $v \succ \bar{w}$, and $v \in \min _{\succeq} T(f)$ implies $f(\bar{w})=0$, hence $f(w)=$ $\rho_{v}(f)(w)=1$. Thus (10) holds. Finally, assume that $w=$ $v(\succ u)$, in which case $u \neq \bar{v}$ by the condition in the lemma. $v \in \min _{\succeq} T(f)$ implies $f(u)=0$, hence $\rho_{v}(f)(u)=0$. Thus (10) again holds.

The following lemma shows how to choose $v$ to be used in $\rho_{v}(f)$ to guarantee that $\rho_{v}(f)$ is regular.
LEMMA 3. Let $f$ be a regular self-dual function of $n(\geq$ 2) variables. If $v \in \min _{\succeq} T(f)$ and $v_{n}=1$, then $\rho_{v}(f)$ is regular.

Proof. By Lemma 2 we have only to show $\bar{v} \nprec v$. We have $f\left(v-e^{(n)}\right)=0$ from $v \in \min _{\succeq} T(f)$. Thus, the selfduality of $f$ implies $f\left(\bar{v}+e^{(n)}\right)=1$, which, together with $v \in \min _{\succeq} T(f)$, in turn implies $\bar{v}+e^{(n)} \nprec v$. It follows from $\bar{v}+e^{(n)} \nprec v$ and $v_{n}=1$ that $\bar{v} \nprec v$.

Interestingly, the existence of $v$ satisfying the condition in Lemma 3 is equivalent the relevance of $x_{n}$ to $f$, as proved in the following Lemma 4.

Lemma 4. For a regular function $f, x_{n}$ is relevant to $f$ if and only if there exists a vector $v \in \min _{\succeq} T(f)$ such that $v_{n}=1$.

Proof. If such a vector $v$ exists, then we have $f(v)=1$ and $f\left(v-e^{(n)}\right)=0$ (by $v \in \min _{\succeq} T(f)$ ). Thus $x_{n}$ is relevant to $f$.

Conversely, if $x_{n}$ is relevant, then there exists a vector $w \in$ $\min T(f)$ such that $w_{n}=1$, since, otherwise, the MDNF of $f$ does not contain variable $x_{n}$, and hence $x_{n}$ is irrelevant. The proof is complete if we show $w \in \min _{\succeq} T(f)$.

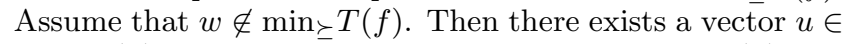
$\min _{\succeq} T(f)$ such that $u \prec w$. Note that $w \in \min T(f)$ and $u \in \min _{\succeq} T(f)$ imply $u \npreceq w-e^{(n)}$. Otherwise, by the profilemonotonicity of $f$, we would have $f\left(w-e^{(n)}\right) \geq f(u)=1$, a contradiction to $w \in \min T(f)$. From $u \npreceq w-e^{(n)}$ and $u \prec w$, it follows that $u_{n}=1$ (possible only if $n \geq 2$ ), implying the only-if part.

Lemma 3 deals with the case where $x_{n}$ is relevant to $f$. To deal with the case where $x_{n}$ is irrelevant to $f$, note that for any $i, j \in V$ such that $i<j$, if $x_{j}$ is relevant to a regular function $f$ then so is $x_{i}$. This implies that $x_{i}$ is relevant to $f$ if and only if $V_{f} \supseteq\{1,2, \ldots, i\}$, in particular, $x_{n}$ is relevant to $f$ if and only if $V_{f}=\{1,2, \ldots, n\}=V$. Corollary 1 below generalizes Lemma 3 to the case where $x_{n}$ may be irrelevant to $f$.

COROLlary 1. Let $f$ be a regular self-dual function such that $\left|V_{f}\right|=i(\geq 2)$. If $v \in \min _{\succeq} T(f)$ and $v_{i}=1$, then $\sigma_{\left(v ; V_{f}\right)}(f)$ is regular.

Proof. Let $I=V_{f}$ in (7). Then $\rho_{v}\left(\operatorname{Proj}_{V_{f}}(f)\right)$ is a regular function on $V_{f}$ by Lemma 3 . This completes the proof, since $\operatorname{Exp}_{V}()$ preserves regularity.

We now have the theoretical foundation for TRANS-REGSD. By Lemma 3 and Corollary 1 , if $x_{n}$ is relevant to a given $f$, we can use transformation $\rho_{v}(f)$, with some $v$, to generate a new regular self-dual function, and repeat this procedure as long as $x_{n}$ is relevant. Once $x_{n}$ becomes irrelevant to the 
newly generated function, $f^{\prime}$, we use $\sigma$ transformations with respect to $V_{f^{\prime}}$, and so forth.

What remains is the discussion of data we need to keep track of in implementing a sequence of $\sigma$ transformations. To represent the sequence of regular self-dual functions $\left\{f^{\prime}\right\}$ that TRANS-REG-SD generates, we represent each such function $f^{\prime}$ in terms of $\min T\left(f^{\prime}\right)$ and $\min _{\succeq} T\left(f^{\prime}\right)$. For a vector $v$, let us introduce the notation, $T_{\succ}(v)^{-}=\{w \mid w \succ v\}$ and $T_{\prec}(v)=\{w \mid w \prec v\}$.

LEMma 5. Let $f$ be a regular self-dual function of $n(\geq 2)$ variables, and let $v \in \min _{\succeq} T(f)$ with $v_{n}=1$. Then we have

$$
\begin{aligned}
& \min T\left(\rho_{v}(f)\right)=\min T(f) \backslash\left(\{ v \} \cup \left\{\bar{v}+e^{(j)} \mid \max O F F\right.\right. \\
&(v)<j \leq n\}) \cup\{\bar{v}\} \\
& \min _{\succeq} T\left(\rho_{v}(f)\right)=\min _{\succeq} T(f) \backslash\left(\{v\} \cup \min _{\succeq} T_{\succ}(\bar{v})\right) \cup\{\bar{v}\} \\
& \cup\left\{u \in \operatorname { m i n } _ { \succeq } T _ { \succ } ( v ) | u \nsucc z \text { for all } z \in \left(\min _{\succeq} T(f) \backslash\right.\right. \\
&\{v\}) \cup\{\bar{v}\}\} .
\end{aligned}
$$

Proof. By (8), we need to consider only the influence of (i) adding $\bar{v}$ to $T(f)$ and (ii) removing $v$ from $T(f)$.

(11) (i): $\bar{v} \in \min T\left(\rho_{v}(f)\right)$ clearly holds. Let us next consider vectors of the form $\bar{v}+e^{(j)}, j \in O N(v)(=O F F(\bar{v}))$, which may cease to be a minimal member as a result of operation (i). Note that these vectors belong to $T(f)$, since $\bar{v} \in \max F(f)$. We claim that $\bar{v}+e^{(j)} \in \min T(f)$ if and only if $j>\max \operatorname{OFF}(v)$. If $j>\max \operatorname{OFF}(v), \bar{v}+e^{(j)} \in \min T(f)$ holds, since otherwise, there exists a vector $w \in \min T(f)$ such that $w<\bar{v}+e^{(j)}$. Since $w \neq \bar{v}$ and $w<\bar{v}+e^{(j)}$, $w \leq \bar{v}+e^{(j)}-e^{(i)}$ holds for some $i \in \operatorname{OFF}(v)(=O N(\bar{v}))$. Since $i<j$, we have $w \prec \bar{v}$. Note that $f(\bar{v})=0$ by the selfduality of $f$. Thus it follows from Lemma 1 that $f(w)=0$ holds, a contradiction to $w \in \min T(f)$. This proves the if part of our claim.

Let $j \in O N(v)(=O F F(\bar{v}))$ with $j \leq \max O F F(v)$, and consider the vector $w=\bar{v}+e^{(j)}-e^{(\max O F F(v))}$. Since $j \in$ $O N(v)$, we have $j<\max O F F(v)$. Thus $w \succ \bar{v}$, and hence $v \succ \bar{w}$ holds. If $f(w)=0$, then $f(\bar{w})=1$ holds by the self-duality of $f$. Since $v \succ \bar{w}$, we have $v \notin \min _{\succeq} T(f)$, a contradiction. Thus $f(w)=1$, implying that $\bar{v}+e^{(j)} \notin$ $\min T(f)$ holds in this case.

(11) (ii): It is clear that $v \notin \min T\left(\rho_{v}(f)\right)$. Note that a vector $u(\neq v) \in T(f)$ satisfying $w \nless u$ for any vector $w \in \min T(f) \backslash\{v\}$ may be in $\min T\left(\rho_{v}(f)\right)$. Such a vector, if any, has the form $u=v+e^{(j)}, j \in O F F(v)$. Since $f(v)=1$, $f\left(v+e^{(j)}-e^{(n)}\right)=1$, i.e., $v+e^{(j)}-e^{(n)} \in T(f)$, follows from the regularity of $f$. Thus no vector of the form $v+$ $e^{(j)}$ belongs to $\min T\left(\rho_{v}(f)\right)$, since, obviously, there exists a $w \in \min T(f)$ satisfying $w \leq v+e^{(j)}-e^{(n)}<v+e^{(j)}, \mathrm{a}$ contradiction.

(12) (i): It is easy to see that $\bar{v} \in \min _{\succeq} T\left(\rho_{v}(f)\right)$ holds, since otherwise, $f(\bar{v})=0$ and there exists a vector $w$ such that $w \prec \bar{v}$ and $f(w)=1$, a contradiction to the regularity of $f$. Consider now any vector $w \in \min _{\succeq} T(f)$ supported by $\bar{v}$, i.e., satisfying $w \succ \bar{v}$. Such a $w$ must be removed from $\min _{\succeq} T(f)$ to construct $\min \succeq T\left(\rho_{v}(f)\right)$. Clearly, each such $w$ is contained in $\min _{\succeq} T_{\succ}(\bar{v})$.

(12) (ii): As noted in (11) (ii), we have $v \notin \min T\left(\rho_{v}(f)\right), a$ fortiori, $v \notin \min _{\succeq} T\left(\rho_{v}(f)\right)$. Let us consider the vectors in $\min _{\succeq} T_{\succ}(v)$, since, besides $\bar{v}$, only they may be contained in

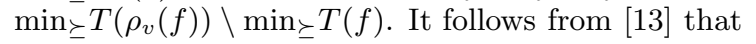

$$
\min _{\succeq} T_{\succ}(v)=\left\{\begin{array}{c}
\left\{v+e^{(n)}\right\} \cup\left\{v+e^{(j)}-e^{(j+1)} \mid v_{j}=\right. \\
\left.0, v_{j+1}=1,1 \leq j \leq n-1\right\} \text { if } v_{n}=0 \\
\left\{v+e^{(j)}-e^{(j+1)} \mid v_{j}=0, v_{j+1}=1,1\right. \\
\leq j \leq n-1\} \text { if } v_{n}=1,
\end{array}\right.
$$

Note that a vector $u \in \min _{\succeq} T_{\succ}(v)$ belongs to $\min _{\succeq} T\left(\rho_{v}(f)\right)$, provided there is no vector $z \in \min _{\succeq} T(f) \backslash\{v\} \cup\{\bar{v}\}$ such that $z \prec u$. This is because $\min _{\succ} T(\bar{f}) \backslash\left(\{v\} \cup \min _{\succ} T_{\succ}(\bar{v})\right) \cup$ $\{\bar{v}\} \subseteq \min _{\succeq} T\left(\rho_{v}(f)\right)$ (see (11) (i) above) and all vectors in $\min _{\succeq} T_{\succ}(\bar{v})$ are supported by $\bar{v}$.

From the proof of Lemma 5 (case (11)(i)), we can see that $\bar{v}+e^{(n)} \in \min T(f)$. Since $v_{n}=1$ implies $n>\max O F F(v)$, $\left\{\bar{v}+e^{(j)} \mid \max O F F(v)<j \leq n\right\}$ is non-empty, and (11) implies Lemma 6.

LEMma 6. Let $f$ be a regular self-dual function of $n(\geq 2)$ variables, and let $v \in \min _{\succeq} T(f)$ with $v_{n}=1$. Then

$$
\begin{aligned}
\left|\min T\left(\rho_{v}(f)\right)\right| & \leq|\min T(f)|-1, \\
\min T\left(\rho_{v}(f)\right)_{n} \cup\left\{v, \bar{v}+e^{(n)}\right\} & =\min T(f)_{n},
\end{aligned}
$$

where $S_{n}$ denotes the set $\left\{v \in S \mid v_{n}=1\right\}$.

We are now ready to describe the transformation algorithm. If we repeatedly apply $\rho_{v}$ operations (with different $v$ 's, of course) to a regular self-dual function $f$, until there is no vector $v \in \min _{\succeq} T(f)$ with $v_{n}=1$, then by Lemmas 3,4 and 6 , we have a regular self-dual function $f^{\prime}$, to which $x_{n}$ is irrelevant. Note that $f^{\prime}$ may not be unique, i.e., it in general depends on the sequence of vectors $v \in \min _{\succeq} T(f)$ with $v_{n}=1$ that are used in $\rho_{v}$.

Now $V_{f^{\prime}}=\left\{1,2, \ldots, j_{1}\right\}$ holds for some $j_{1} \leq n-1$. If $j_{1}=1$, we have $f^{\prime}=x_{1}$ and we are done. If $j_{1} \neq 1$, on the other hand, we apply $\sigma_{\left(v ; V_{f^{\prime}}\right)}$ operations to $f^{\prime}$ instead of $\sigma_{\left(v ; V_{f}\right)}\left(=\rho_{v}\right)$, until there is no vector $v \in \min _{\succeq} T\left(f^{\prime}\right)$ with $v_{j_{1}}=1$. Since all the lemmas presented in this section are still valid for $\sigma_{\left(v ; V_{f^{\prime}}\right)}$ and $v_{j_{1}}=1$ in place of $\sigma_{\left(v ; V_{f}\right)}\left(=\rho_{v}\right)$ and $v_{n}=1$, we obtain a regular self-dual function $f^{\prime \prime}$, whose relevant variable set is $V_{f^{\prime \prime}}=\left\{1,2, \ldots, j_{2}\right\}$ with $j_{2}<j_{1}$. By repeating this argument, we reach the 1-variable regular selfdual function $x_{1}$. Formally, this sequence of transformations can be stated as follows.

\section{Algorithm TRANS-REG-SD}

Input: $\min T(f)$, where $f$ is a regular self-dual function.

Output: Regular self-dual functions $f_{0}(=f), f_{1}, f_{2}, \ldots$, $f_{m}\left(=x_{1}\right)$.

Step 0: Let $i=0$ and $f=f_{0}$.

Step 1: Output $f_{i}$. If $f_{i}=x_{1}$, then halt. 
Step 2: $f_{i+1}=\sigma_{\left(v^{(i)} ; V_{f_{i}}\right)}\left(f_{i}\right)$, where $v^{(i)} \in \min _{\succeq} T\left(f_{i}\right)$ and $v_{\max V_{f_{i}}}^{(i)}=1$. $i:=i+1$. Return to Step 1 .

By (14), the number $m$ in the output from TRANS-REG-SD satisfies $m \leq|\min T(f)|-1$. Since every self-dual function $f$ satisfies $\rho_{\bar{v}}\left(\rho_{v}(f)\right)=f$ (see $(8)$ ), we can transform $x_{1}$ into any regular self-dual function $g$ by repeatedly applying $\sigma$ operations to $x_{1}$ at most $|\min T(g)|-1$ times. Thus we have the following theorem.

THEOREM 2. Let $f$ and $g$ be any two regular self-dual functions. Then $f$ can be transformed into $g$ by repeatedly applying $\sigma$ operations to $f$ at most $|\min T(f)|+|\min T(g)|-$ 2 times.

In the subsequent sections, we study some applications of algorithm TRANS-REG-SD.

\section{OPTIMUM SELF-DUAL FUNCTION FOR REGULAR FUNCTIONAL $\boldsymbol{\Phi}$}

Let $\varphi$ be a pseudo Boolean function, i.e., $\varphi$ is a mapping from $\{0,1\}^{n}$ to the set of real numbers $\mathbb{R}$. $\varphi$ is said to be g-regular if it is profile-monotone, i.e., $\varphi(v) \geq \varphi(w)$ holds for all pairs of vectors $v$ and $w$ with $v \succ w$. Define a functional $\Phi()$ of Boolean functions $f$ as follows:

$$
\Phi(f)=\sum_{v \in T(f)} \varphi(v),
$$

where $\varphi$ is a pseudo Boolean function. $\Phi$ is also said to be $\mathrm{g}$ regular if $\varphi$ is g-regular. As an example of a g-regular pseudo Boolean functional of interest, we cite the availability $A(f)$ of a Boolean function $f$. Assume that each element $i \in V$ has the operational probability $p_{i}\left(0 \leq p_{i} \leq 1\right)$, i.e., the $i$-th element is operational with probability $p_{i}$. We also assume that the probabilities for different elements are independent. Then the availability of a Boolean function $f$ is defined by

$$
A(f)=\sum_{v \in T(f)}\left(\prod_{i \in O N(v)} p_{i} \prod_{i \in O F F(v)}\left(1-p_{i}\right)\right) .
$$

If we interpret $T(f)$ as the set of states in which the $n$ element system defined by the Boolean function $f$ is working, then $A(f)$ represents the probability that the system represented by $f$ is working. Availability has been studied extensively, especially, in the case where $f$ represents a ND coterie (i.e., $f$ is positive self-dual) $[1,4,7,16,17,19]$. As commented in the Introduction, we can assume without loss of generality that

$$
p_{1} \geq p_{2} \geq \ldots \geq p_{n} \geq 1 / 2 .
$$

Now, let $\varphi(v)=\prod_{i \in O N(v)} p_{i} \prod_{i \in O F F(v)}\left(1-p_{i}\right)$. Then we have $\Phi(f)=A(f)$. It follows from the assumption on the order of probabilities that $A(f)$ is g-regular.

In this section, we consider the functions $f$ that maximize g-regular functional $\Phi$ among all self-dual functions.

Lemma 7. Given a g-regular function $\varphi$, let $\Phi$ be a $g$ regular functional defined by (16). Then the following statements regarding $f$ are equivalent. (i) $\Phi(f)$ is maximum among all self-dual functions.

(ii) All vectors $v \in T(f)$ satisfy $\varphi(v) \geq \varphi(\bar{v})$.

(iii) All vectors $v \in \min _{\succeq} T(f)$ satisfy $\varphi(v) \geq \varphi(\bar{v})$.

Proof. Let us prove (i) $\Longrightarrow$ (ii) $\Longrightarrow$ (iii) $\Longrightarrow$ (i). Since (ii) $\Longrightarrow$ (iii) is obvious, we show (i) $\Longrightarrow$ (ii) and (iii) $\Longrightarrow$ (i).

(i) $\Longrightarrow$ (ii): If there exists a vector $v \in T(f)$ such that $\varphi(v)<\varphi(\bar{v})$, then the function $g$ defined by $T(g)=(T(f) \backslash$ $\{v\}) \cup\{\bar{v}\}$ satisfies $\Phi(g)>\Phi(f)$. Since $g$ is self-dual, $\Phi(f)$ is not maximum among all self-dual functions.

(iii) $\Longrightarrow$ (i): Assume that $\Phi(f)$ is not maximum among all self-dual functions. Then there exists a self-dual function $g$ such that $\Phi(g)>\Phi(f)$. Since $\Phi(g)>\Phi(f)$, some vector $v \in T(f) \backslash T(g)$ satisfies $\varphi(v)<\varphi(\bar{v})$. For this $v$, let $w$ be a vector in $\min _{\succeq} T(f)$ such that $w \preceq v$. By the g-regularity of $\varphi, \varphi(w) \leq \varphi(v)$ holds. Moreover, since $\bar{w} \succeq \bar{v}$ by $w \preceq v$, $\varphi(\bar{w}) \geq \varphi(\bar{v})$ holds. Thus, we have

$$
\varphi(w) \leq \varphi(v)<\varphi(\bar{v}) \leq \varphi(\bar{w}) .
$$

This means that this $w$ satisfies $w \in \min _{\succeq} T(f)$ and $\varphi(w)<$ $\varphi(\bar{w})$.

THEOREM 3. Let $\Phi(f)$ be a g-regular functional defined by (16). Then there exists a regular self-dual function $f$ which maximizes $\Phi(f)$ among all self-dual functions.

Proof. Let $f$ be a regular self-dual function that maximizes $\Phi$ among all regular self-dual functions. We claim that $f$ in fact maximizes $\Phi$ among all self-dual functions. If not, by Lemma 7 , there exists a vector $v \in \min _{\succeq} T(f)$ such that $\varphi(v)<\varphi(\bar{v})$. Note that $v \nsucceq \bar{v}$ holds, since, otherwise (i.e., $v \succeq \bar{v}), \varphi(v) \geq \varphi(\bar{v})$, a contradiction. Thus, it follows from Lemma 2 that $\rho_{v}(f)$ is regular and self-dual. Moreover, by Eq. (8), we have $\Phi\left(\rho_{v}(f)\right)>\Phi(f)$, which contradicts the assumption.

However, there may be non-regular functions $f$ that also maximize $\Phi(f)$.

Based on Theorem 3, the following algorithm computes an optimum regular self-dual function.

\section{Algorithm OPT-REG-SD}

Input: A membership oracle of g-regular function $\varphi$.

Output: A regular self-dual function $f$ that maximizes $\Phi(f)$ among all self-dual functions.

Step 0: Let $i:=1$ and $f:=x_{1}$.

Step 1: While $\exists v \in \min _{\succeq} T(f)$ such that $v_{i}=0, v\left[V_{i}\right] \nsucceq$ $\bar{v}\left[V_{i}\right]$ and $\varphi\left(v^{\prime}\right)<\varphi\left(\overline{v^{\prime}}\right)$ for $v^{\prime}=v+\sum_{j=i+1}^{n} e^{(j)}$, do $f:=$ $\sigma_{\left(v ; V_{i}\right)}(f)$, where $V_{i}=\{1,2, \ldots, i\}$.

Step 2: If $i=n$, output $f$ and halt. Otherwise, let $i:=i+1$ and return to Step 1. 
Note that the set $\min _{\succeq} T(f)$ in the while statement of Step 2 is updated as a result of applying the $\sigma$ transformation to $f$ in Step 2.

EXAMPLE 2. Let us consider the availability of the function on 6 variables, when $p_{1}=9 / 10, p_{2}=6 / 7, p_{3}=4 / 5$, $p_{4}=7 / 10$ and $p_{5}=3 / 5$. Recall that $\Phi(f)=A(f)$ is given by Eq. (17). We apply Algorithm OPT-REG-SD to this $\Phi(f)$.

Step 0: Let $i:=1, f:=x_{1}\left(\right.$ thus, $\left.\min _{\succeq} T(f)=\{(10000)\}\right)$, and $u=(10000)$.

First iteration of Steps 1-2: Since $u_{1}=1$, skip Step 1. Step 2 sets $i:=2$.

Second iteration of Steps 1-2: Since $u\left[V_{2}\right]=(10) \succeq$ $\bar{u}\left[V_{2}\right]=(01)$ for the only vector $u$ in $\min _{\succeq} T(f)$, skip Step 1. Step 2 sets $i:=3$.

Third iteration of Steps 1-2: Vector $u \in \min _{\succeq} T(f)$ satisfies $u_{3}=0, u\left[V_{3}\right]=(100) \nsucceq \bar{u}\left[V_{3}\right]=(011)$, but $\varphi(10011)=9 / 10 \times 1 / 7 \times 1 / 5 \times 7 / 10 \times 3 / 5=189 / 17500>$ $\varphi(01100)=1 / 10 \times 6 / 7 \times 4 / 5 \times 3 / 10 \times 2 / 5=144 / 17500$.

(If we were to apply $\sigma_{\left(u ; V_{3}\right)}$ to $f$, we would have $\Phi\left(\sigma_{\left(u ; V_{3}\right)}(f)\right.$ $\Phi(f)$.) Thus skip Step 1. Step 2 sets $i:=4$.

Fourth iteration of Steps 1-2: Vector $u$ satisfies $u_{4}=$ $0, u\left[V_{4}\right]=(1000) \nsucceq \bar{u}\left[V_{4}\right]=(0111)$. Moreover, we have $\varphi(10001)<\varphi(01110)$, since $\varphi(10001)=9 / 10 \times$ $1 / 7 \times 1 / 5 \times 3 / 10 \times 3 / 5=81 / 17500$ and $\varphi(01110)=$ $1 / 10 \times 6 / 7 \times 4 / 5 \times 7 / 10 \times 2 / 5=336 / 17500$. Thus $f$ is transformed to

$$
f:=\sigma_{\left(u ; V_{4}\right)}(f)=12+13+14+234 .
$$

For this new $f$, we have $\min _{\succeq} T(f)=\{u=(10010), v=$ (01110)\}. Since $u_{4}=v_{4}=1$, we skip Step 1. Step 2 sets $i:=5$.

Fifth iteration of Steps 1-2: Vector $u$ satisfies $u_{5}=0$, $u\left[V_{5}\right]=(10010) \nsucceq \bar{u}\left[V_{5}\right]=(01101)$. Moreover, we have $\varphi(10010)<\varphi(01101)$, since $\varphi(10010)=9 / 10 \times$ $1 / 7 \times 1 / 5 \times 7 / 10 \times 2 / 5=126 / 17500$ and $\varphi(01101)=$ $1 / 10 \times 6 / 7 \times 4 / 5 \times 3 / 10 \times 3 / 5=216 / 17500$. Thus $f$ is transformed to

$$
\begin{aligned}
f:=\sigma_{\left(u ; V_{5}\right)}(f)= & 12+13+14(2+3+5) \\
& +234+235 \\
= & 12+13+145+234+235 .
\end{aligned}
$$

As a result, we have $\min _{\succeq} T(f)=\{u=(10100), v=$ $(10011), w=(01101)\}$. Since $v_{5}=w_{5}=1$, we need to consider only $u=(10100)$. Since $u_{5}=0, u\left[V_{5}\right]=$ $(10100) \nsucceq \bar{u}\left[V_{5}\right]=(01011)$, but $\varphi(10100)=216 / 17500$ $>\varphi(01011)=126 / 17500$, skip Step 1. Since $i=n=$ 5 , output function $f$ given by (18) and halt.

Let $f_{i}, i=1,2, \ldots, n$, be the function $f$ after the $i$-th iteration of Step 1 of OPT-REG-SD has been completed. Then clearly $V_{f_{i}} \subseteq V_{i}(=\{1,2, \ldots, i\})$ holds. Moreover, we have the following lemma:

Lemma 8. Let $f_{i}, i=1,2, \ldots, n$, be as defined above. For each $i=1,2, \ldots, n$, all vectors $v \in \min _{\succeq} T\left(f_{i}\right)$ with $v\left[V_{i}\right] \nsucceq \bar{v}\left[V_{i}\right]$ satisfy

$$
\varphi\left(v^{\prime}\right) \geq \varphi\left(\overline{v^{\prime}}\right)
$$

where $v^{\prime}=v+\sum_{j=i+1}^{n} e^{(j)}$.
Proof. If $i=1$, then $f_{1}=x_{1}$, and the lemma holds in this case, since $\min _{\succeq} T\left(f_{1}\right)=\{v=(100 \cdots 0)\}$ and $v\left[V_{1}\right] \succeq$ $\bar{v}\left[V_{1}\right]$.

Assuming it holds for $i=k$, consider the case where $i=$ $k+1$. Let us consider the vector $v \in \min _{\succ} T\left(f_{k+1}\right)$ with $v\left[V_{k+1}\right] \succeq \bar{v}\left[V_{k+1}\right]$. If $v_{k+1}=0$, then $v$ satisfies $\varphi\left(v^{\prime}\right) \geq$ $\varphi\left(\overline{v^{\prime}}\right)$, where $v^{\prime}=v+\sum_{j=k+2}^{n} e^{(j)}$, since, otherwise, $v$ would have been removed from $T\left(f_{k+1}\right)$ in Step 1 by applying the operation $\sigma_{\left(v ; V_{k+1}\right)}$ to $f_{k+1}$. This contradicts the definition of $f_{k+1}$.

If $v_{k+1}=1$, on the other hand, there are two possibilities: (1) $f_{k}(v)=0$ and $(2) f_{k}(v)=1$. In case (1), since $v \in$ $T\left(f_{k+1}\right) \backslash T\left(f_{k}\right), f$ was updated by $f:=\sigma_{\left(\overline{v^{\prime}} ; V_{k+1}\right)}(f)$ in the $(k+1)$-st iteration of Step 1. Therefore, we have $\varphi\left(v^{\prime}\right) \geq$ $\varphi\left(\overline{v^{\prime}}\right)$.

In case $(2)$, assuming $\varphi\left(v^{\prime}\right)<\varphi\left(\overline{v^{\prime}}\right)$, we derive a contradiction. $V_{f_{k}} \subseteq V_{k}$ implies $f_{k}\left(v-e^{(k+1)}\right)=1$. Since $v\left[V_{k+1}\right] \nsucceq$ $\bar{v}\left[V_{k+1}\right]$, we have $\left(v-e^{(k+1)}\right)\left[V_{k}\right] \nsucceq\left(\overline{v-e^{(k+1)}}\right)\left[V_{k}\right]$. Note that $\left(v-e^{(k+1)}\right)^{\prime}=v-e^{(k+1)}+\sum_{j=k+1}^{n} e^{(j)}=v^{\prime}$. Thus, if $v-e^{(k+1)} \in \min _{\succeq} T\left(f_{k}\right)$, by assumption, $f_{k}$ would have been updated by $f_{k}:=\sigma_{\left(v-e^{(k+1)} ; V_{k}\right)}\left(f_{k}\right)$. This contradicts the definition of $f_{k}$. Now, since $v-e^{(k+1)} \notin \min \succ T\left(f_{k}\right)$, there exists a vector $w \in \min \succeq T\left(f_{k}\right)$ such that $w \preceq v-e^{(k+1)}$. We can easily see that this $w$ satisfies $w\left[V_{k}\right] \nsucceq \bar{w}\left[V_{k}\right]$ and $\varphi\left(w^{\prime}\right)<\varphi\left(\overline{w^{\prime}}\right)$, where $w^{\prime}=w+\sum_{j=k+1}^{n} e^{(j)}$. This implies that $f_{k}$ would have been updated by $f_{k}:=\sigma_{\left(w ; V_{k}\right)}\left(f_{k}\right)$, again a contradiction.

Lemma 9. Let $f_{n}$ be as defined above. Then $f_{n}$ maximizes $\Phi$ among all self-dual functions.

Proof. Lemma 8 asserts (when $i=n$ ) that all vectors $v \in \min _{\succeq} T\left(f_{n}\right)$ with $v \nsucceq \bar{v}$ satisfy $\varphi(v) \geq \varphi(\bar{v})$. As for vectors $v \in \min _{\succ} T\left(f_{n}\right)$ with $v \succeq \bar{v}$, the g-regularity of $\varphi$ implies $\varphi(v) \geq \varphi(\bar{v})$. Together with Lemma 7, this completes the proof.

Therefore, OPT-REG-SD computes an optimum function $f\left(=f_{n}\right)$. Moreover, it requires polynomial time in $n$ and the size of $f$. (Due to the space limitation, we omit the proof. See [13]).

THEOREM 4. Algorithm OPT-REG-SD correctly outputs a regular self-dual function $f$ that maximizes $\Phi$ among all self-dual functions in $O\left(n^{3}|\min T(f)|\right)$ time.

\section{GENERATION OF ALL REGULAR ND COTERIES}

Let $\mathcal{C}_{R-S D}(n)$ denote the class of all regular self-dual functions of $n$ variables. We present in this section an algorithm to generate all functions in $\mathcal{C}_{R-S D}(n)$ by applying the operator $\sigma$. The algorithm is incrementally polynomial [11] in the sense that the $i$-th function $\phi_{i} \in \mathcal{C}_{R-S D}(n)$ is output in polynomial time in $n$ and $\sum_{j=0}^{i-1}\left|\min T\left(\phi_{j}\right)\right|$, for $i=1,2, \ldots,\left|\mathcal{C}_{R-S D}\right|$. 
To visualize the algorithm, we first define an undirected graph $G_{n}=\left(\mathcal{C}_{R-S D}(n), E\right)$, where $(g, f) \in E$, if there exists a vector $v \in \min _{\succeq} T(g)$ such that $\sigma_{(v ; I)}(g)=f$ for some $I \supseteq V_{g}$. Figure 1 shows the graph $G_{5}$. (Ignore the arrows on some edges).

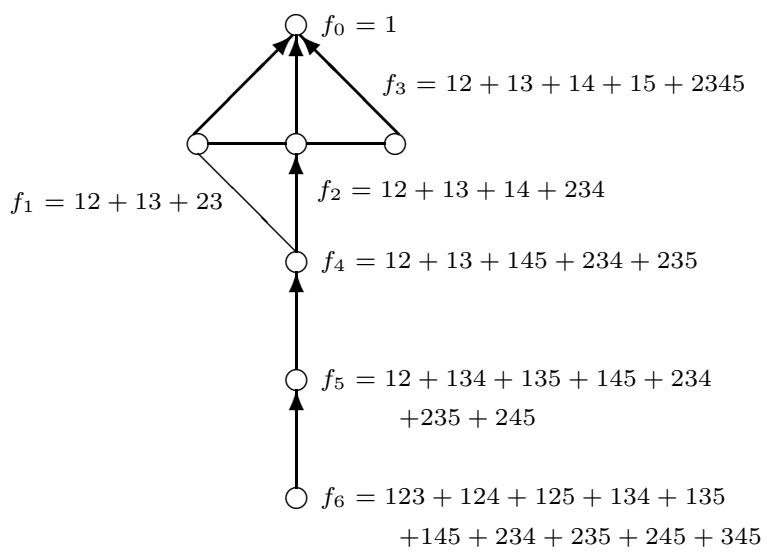

Figure 1: The graph $G_{5}$.

Theorem 2 implies that $G_{n}$ is connected. Moreover, the condition $(g, f) \in E$ holds if and only if $(f, g) \in E$, i.e., $G_{n}$ is undirected. Let $f_{0}=x_{1}$ be the designated function in $\mathcal{C}_{R-S D}(n)$, and consider the problem of transforming an arbitrary function $g \in \mathcal{C}_{R-S D}(n)$ to $f_{0}$ by repeatedly applying operation $\sigma$ in Algorithm TRANS-REG-SD. Note that the transformation path from a given $g$ to $f_{0}$ is not unique. Thus, to make the path unique, we choose for each $\sigma$ operation the lexicographically smallest vector $\tilde{v} \in \min _{\succeq} T(g)$ such that $\tilde{v}_{\max V_{g}}=1 .^{3}$ Let $\mu$ be such an operation, i.e.,

$$
\mu(g)=\sigma_{\left(\tilde{v} ; V_{g}\right)}(g) .
$$

In this way, we define a directed spanning tree of $G_{n}, R T=$ $\left(\mathcal{C}_{R-S D}(n), A_{R T}\right)$, such that $(g, f)$ is a directed arc in $A_{R T}$ if and only if $\mu(g)=f$. Clearly, this $R T$ is an in-tree rooted at $f_{0}=x_{1}$. In Figure $1, A_{R T}$ is indicated by the thick arcs.

Our algorithm will traverse $R T$ from $f_{0}$ in a depth-first manner, outputting each regular function $f$ when it first visits $f$. This type of enumeration is called reverse search in [2]. When $R T$ is traversed from $f_{0}$, for each arc $(g, f) \in A_{R T}$, the end node $f$ is visited first, i.e., before $g$. Unfortunately, at $f$ we cannot distinguish between the arcs in $A_{R T}$ and the edges in $E$ of $G_{n}$. In other words, knowing $f$, we cannot find $g$ such that $(g, f) \in A_{R T}$. Note that (20) computes $f$ given $g$, not the other way around. In Lemma 10 below, we find the "inverse" of (20) in the sense that we find the conditions on the choice of $w \in \min _{\succeq} T(f)$ such that $g=\sigma_{\left(w ; V_{g}\right)}(f)$.

For a vector $v \in\{0,1\}^{n}$ and $I \subseteq V$, let $v[I] \underline{0}$ denote the vector $u$ defined by $O N(u)=O F F(v) \cap I$, i.e., $u[I]=v[I]$ and the remaining components of $u$, if any, are all set to 0 's.

LEMmA 10. Let $f \in \mathcal{C}_{R-S D}(n)$ and $g=\sigma_{\left(w ; V_{g}\right)}(f)$ for $w \in \min _{\succeq} T(f)$ such that $\bar{w}\left[V_{g}\right] \nprec w\left[V_{g}\right]$ and $V_{g} \supseteq V_{f}$. Then

\footnotetext{
${ }^{3}$ For example, the lexicographic order of all vectors in $\{0,1\}^{3}$ is $(000),(001),(010),(011),(100),(101),(110),(111)$.
}

$f=\mu(g)\left(=\sigma_{\left(\tilde{v} ; V_{g}\right)}(g)\right)$ if and only if

(a) $w_{\max } V_{g}=0$,

(b) $w_{1}=1$, and

(c) $\bar{w}\left[V_{g}\right] \underline{0}$ is lexicographically the smallest among all vectors $u \in \min _{\succeq} T(f)$ with $u_{\max } V_{g}=1$.

Proof. Let us first consider the only-if part, assuming $f=\sigma_{\left(\tilde{v} ; V_{g}\right)}(g)$. Then $g=\sigma_{\left(w ; V_{g}\right)}(f)$ implies that $w=$ $\overline{(\tilde{v})}\left[V_{g}\right] \underline{0}$. Since $\tilde{v}_{\max V_{g}}=1$ by definition, we have $w_{\max V_{g}}=$ 0 , hence (a) holds. Thus, it follows from (12) and (13) that

$$
\begin{aligned}
& \min _{\succeq} T(g)_{x_{\max V_{g}}=1}=
\end{aligned}
$$

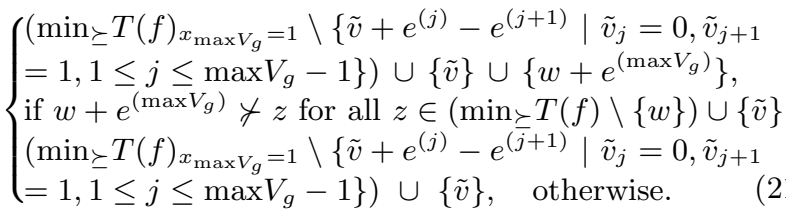

where $S_{x_{i}=1}$ denotes the set $\left\{v \in S \mid v_{i}=1\right\}$. Now, by definition, $\tilde{v}$ is the lexicographically smallest vector in

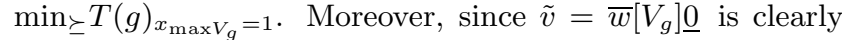
lexicographically smaller than $u=\tilde{v}+e^{(j)}-e^{(j+1)}$ with $\tilde{v}_{j}=0$ and $\tilde{v}_{j+1}=1,(21)$ implies (c).

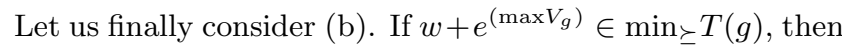
$w_{1}=1$ holds by (21), since $\tilde{v}$ is lexicographically smaller than $w+e^{\left(\max V_{g}\right)}$. On the other hand, if $w+e^{\left(\max V_{g}\right)} \notin$ $\min _{\succeq} T(g)$, there exists a vector $u \in \min _{\succeq} T(g)$ such that $u \prec w+e^{\left(\max V_{g}\right)}$ and $u_{\max V_{g}}=1$ (the proof is similar to that of Lemma 4). If $w_{1}=0$, then we have $u_{1}=0$ (by $u \prec w+e^{\left(\max V_{g}\right)}$ ) and $\tilde{v}_{1}=1$ (by $\left.\tilde{v}=\bar{w}\left[V_{g}\right] \underline{0}\right)$. However, this implies that $u$ is lexicographically smaller than $\tilde{v}$, contradicting $\mu(g)=\sigma_{\left(\tilde{v} ; V_{g}\right)}(g)$. Therefore, (b) must hold.

The if part is implied by (21).

Note that, if $V_{g} \neq V_{f}$ (i.e., $V_{g} \supset V_{f}$ ), Lemma 10 implies that $f=\mu(g)$ if and only if $w_{1}=1$, since $V_{g} \supset V_{f}$ and $w \in$ $\min _{\succeq} T(f)$ imply (a). Thus, for an index set $I \supset V_{f}$, every vector $w \in \min _{\succeq} T(f)$ which satisfies $w_{1}=1$ and $\bar{w}[I] \nprec w[I]$ always produces $g=\sigma_{(w ; I)}(f)$ such that $f=\mu(g)$.

Let

$$
\begin{aligned}
M_{\text {sum }} & =\sum_{f \in \mathcal{C}_{R-S D^{(n)}}}|\min T(f)|, \\
M_{\max } & =\max _{f \in \mathcal{C}_{R-S D^{(n)}}}|\min T(f)| .
\end{aligned}
$$

Although the details are omitted due to the space limitation, we have the following results [13].

TheOREM 5. All functions in $\mathcal{C}_{R-S D}(n)$ can be generated in incrementally polynomial time. It requires $O\left(n^{3} \mid \mathcal{C}_{R-S D}\right.$ $\left.(n) \mid+n M_{\text {sum }}\right)$ time and $O\left(n M_{\max }\right)$ space.

Corollary 2. All functions in $\mathcal{C}_{R-S D}(n)$ can be scanned in $O\left(n^{3}\left|\mathcal{C}_{R-S D}(n)\right|\right)$ time. 
We reiterate here that regular functions are all representatives of a permutation class, i.e., no regular function $f$ is equivalent to another regular function $g(\neq f)$ under permutation. Therefore, our algorithm generates non-equivalent functions. Let us remark that the algorithms in $[5,8]$ are not polynomial, if we try to output only non-equivalent functions.

It is known that the positive self-dual functions of up to $n=$ 5 variables are all threshold functions (and hence regular, if we consider the representatives), but there are many nonregular self-dual functions for $n \geq 6$, even if we consider the representatives. Moreover, it is known [14] that all regular self-dual functions for $n \leq 9$ are threshold functions.

\section{CONCLUSION}

We have introduced a new operator $\sigma$, which is similar to the $\rho$ operator used by Bioch and Ibaraki [5]. It transforms a non-dominated (ND) coterie to another ND coterie. We showed that any regular ND coterie can be transformed to any other regular ND by a sequence of $\sigma$ operations, and tried to find the shortest such sequence. As another application of the $\sigma$ operation, we presented an incrementallypolynomial-time algorithm for generating all regular ND coteries, and showed that we can construct an optimum coterie $C$ with respect to a "g-regular" function in polynomial time.

The challenging problem of deciding whether a given coterie is ND is still open.

\section{ACKNOWLEDGMENTS}

We thank the referees for their helpful suggestions.

\section{REFERENCES}

[1] Y. Amir and A. Wool, Optimal availability quorum systems: theory and practice, Information Processing Letters, 65 (1998) 223-228.

[2] D. Avis and K. Fukuda, Reverse search for enumeration, Discrete Applied Mathematics, 65 (1996) 21-46.

[3] D. Barbara and H. Garcia-Molina, The vulnerability of vote assignment, ACM Trans. on Computer Systems, 4 (1986) 187-213.

[4] D. Barbara and H. Garcia-Molina, The reliability of voting mechanisms, IEEE Trans. on Computers, 36 (1987) 1197-1208.

[5] J. C. Bioch and T. Ibaraki, Generating and approximating positive non-dominated coteries, IEEE Transactions on Parallel and Distributed Systems, 6 (1995) 905-914.

[6] S.B. Davidson, Replicated data and partition failures, in: S. Mullender (ed.), Distributed Systems, Ch.13, Addison Wesley, 1989.

[7] K. Diks, E. Kranakis, K. Krizanc, B. Mans and A. Pelc, Optimal coteries and voting schemes, Information Processing Letters, 51 (1994) 1-6.

[8] H. Garcia-Molina and D. Barbara, How to assign votes in a distributed system, J. of the ACM, 32 (1985) 841-860.
[9] D.K. Gifford, Weighted voting for replicated data, Proc. the 7th Symposium on Operating System Princiles, Pacific Grove, Calif., Dec. 1979, 150-162, ACM, New York.

[10] T. Ibaraki and T. Kameda, Theory of coteries: Mutual exclusion in distributed systems, IEEE Trans. on Parallel and Distributed Syst., 4 (1993) 779-794.

[11] D.S. Johnson, M. Yannakakis and C.H. Papadimitriou, On generating all maximal independent sets, Information Processing Letters, 27 (1988) 119-123.

[12] L. Lamport, Time, clocks, and the ordering of events in a distributed system, Comm. of the ACM, 21 (1978) 558-565.

[13] K. Makino and T. Kameda, Transformation of Regular Non-Dominated Coteries, DIMACS Technical Reports 99-41, 1999.

[14] S. Muroga, Threshold Logic and Its Applications, Wiley-Interscience, 1971.

[15] M. Naor and A. Wool, Access control and signatures via quorum secret sharing, IEEE Trans. on Parallel and Distributed Syst., 9 (1998) 909-922.

[16] D. Peleg and A. Wool, The availability of quorum systems, Information and Computation 123 (1995) $210-223$.

[17] M. Spasojevic and P. Berman, Voting as the optimal static pessimistic scheme for managing replicated data, IEEE Trans. on Parallel and Distributed Syst. 5 (1994) 64-73.

[18] R.H. Thomas, A majority consensus approach to concurrency control, ACM Trans. on Database Syst., 4 (1979) 180-209.

[19] Z. Tong and R.Y. Kain, Vote assignments in weighted voting mechanisms, Proc. 7th Symp. on Reliable Distributed Systems, Oct. 1988, 138-143. 